\title{
Zuschläge und Multiplikatoren in der GOÄ nicht vergessen
}

\begin{tabular}{|r|c|l|l|}
\hline \multicolumn{2}{|l|}{ Tabelle 2} & Euro \\
\hline GOÄ & Faktor & Legende & 10,73 \\
\hline 1 & 2,3 & Beratung z.B. telefonisch & 16,32 \\
\hline 1 & 3,5 & Eingehende Beratung < 10 Minuten & 20,11 \\
\hline 3 & 2,3 & Beratung mindestens 10 Minuten & 30,60 \\
\hline 3 & 3,5 & Beratung < 20 Minuten & 40,22 \\
\hline 5 & 2,3 & Eingehende Beratung mindestens 20 Minuten & 10,73 \\
\hline 5 & 2,3 & Klinische Untersuchung ein Organsystem & 16,32 \\
\hline 7 & 2,5 & Klinische Untersuchung mehrere Organgsysteme & 21,45 \\
\hline 8 & 2,5 & Vollständige Untersuchung ein Organsystem & 32,64 \\
\hline Einsatz des Multiplikators bei GoÄ-Leistungen. & 34,86 \\
\hline
\end{tabular}

— Die Honorierung von Leistungen zu besonderen Zeiten ist im Einheitlichen Bewertungsmaßstab (EBM) und der Gebührenordnung für Ärzte (GOÄ) unterschiedlich geregelt. Viele Praxen vergessen deshalb insbesondere bei der Privatabrechnung die hierfür vorgesehenen Zuschläge in den Abschnitten B II und B V. Anders als im EBM gibt es in der GOÄ keine speziellen Ziffern für diese Zuschläge, sondern Buchstaben, die auch noch auf ganz bestimmte Weise mit den korrespondierenden GOÄLeistungen kombiniert werden müssen.

Im Abschnitt B II stehen die „Zuschläge zu Beratungen und Untersuchungen nach den Nummern 1, 3, 4, 5, 6, 7 oder 8." Das bedeutet, dass z. B. im Falle einer Inanspruchnahme um 21:00 Uhr wegen Atemwegsbeschwerden mit Fieber die Nrn. 1 und 7B GOÄ berechnet werden können. Beachtenswert ist dabei, dass die Zuschläge für die besonderen Inanspruchnahmen in der GOÄ nur mit dem einfachen Faktor berechnungsfähig sind. Der Zuschlag B gilt von 20 Uhr bis 22 Uhr und morgens von 6 Uhr bis 8 Uhr. Den Zuschlag C kann man von $22 \mathrm{Uhr}$ bis 6 Uhr berechnen, und am Wochenende kommt der Zuschlag $D$ dazu. Dabei spielt es keine Rolle, ob der Patient bestellt war oder nicht. Nur im Falle von regulär abgehaltenen Sprechstunden am Samstagvormittag ist der Zuschlag D nur mit halbem Faktor berechnungsfähig.

Die Zuschläge A bis D können auch für telefonische Inanspruchnahmen berechnet werden. Dabei gilt es aber, einige Ausschlussregelungen zu beachten. Der Zuschlag $A$ ist neben den Zuschlägen $B, C$ und/oder $D$ nicht berechnungsfähig. Neben dem Zuschlag C ist der Zuschlag B nicht berechnungsfähig. Zuschlag $K 1$ ist neben den Zuschlägen $A, B, C$ und/oder $D$ immer berechnungsfähig. Neben den Zuschlägen $A$, $B, C, D$ und/oder $K 1$ sind die Zuschläge $E, F$, $G, H, K 2$ nicht berechnungsfähig. Für Inanspruchnahmen am Wochenende ist der Zuschlag D mit den Zuschlägen B oder C kombinierbar.

Die Zuschläge für die Besuche, Visiten, Konsiliartätigkeit und Assistenz nach den Nrn. 45 bis 62 GOÄ regelt Abschnitt B V. Hier gelten die Zuschläge E bis J sowie K2. Der Zu- schlag E ist dabei neben den Zuschlägen F, $\mathrm{G}$ und/oder $\mathrm{H}$ nicht berechnungsfähig. Der Zuschlag $\mathrm{E}$ ist neben Leistungen nach Nr. 45 und/oder 46 nicht berechnungsfähig. Die Zuschläge $F, G, H$ sind neben den Leistungen nach den Nrn. 45, 46, 48 und 52 nicht berechnungsfähig. Zuschlag $\mathrm{G}$ ist neben den Leistungen nach den Nummern 45, 46, 48 und 52 nicht berechnungsfähig. Neben dem Zuschlag nach Buchstabe $G$ ist der Zuschlag nach Buchstabe F nicht berechnungsfähig. Für Leistungen am Wochenende hingegen ist der Zuschlag $\mathrm{H}$ mit den Zuschlägen F oder G kombinierbar.

\section{MMW Kommentar}

Ähnlich wie bei den Unzeit-Zuschlägen ist in der GOÄ die Situation beim Einsatz des Multiplikators zu sehen (siehe Tabelle 2). Auch hier wird sicherlich oft Honorar verschenkt, weil der dort ausdrücklich vorgesehene Steigerungsfaktor in Abhängigkeit von Schwierigkeit, Umständen und Zeitfaktor bei der Ausführung der Leistungen zu wenig Beachtung findet. Dies gilt insbesondere unter dem Aspekt, dass die GOÄ schon lange renovierungsbedürftig ist und gerade die sog. "Sprechende Medizin" kaum leistungsgerecht abbildet. Bis eine neue GOÄ in Kraft tritt, die diesen Bereich besser berücksichtigt, kann man sich aber durch den differenzierten Einsatz des jetzt noch vorhandenen Multiplikators helfen. Dessen Einsatz ist insbesondere unter Berücksichtigung des Zeitaufwandes speziell bei Gesprächsleistungen und Untersuchungen in der Lage, den z. T. erheblichen Bewertungsabstand zwischen den betreffenden Gebührenordnungspositionen zu reduzieren. In der Tabelle sind Beispiele für eine solche differenzierte Abrechnung der Leistungen dargestellt, wobei diese Auflistung keinen Anspruch auf Vollständigkeit erhebt. 